\title{
Haematology
}

\section{A TWIST OF FATE - LINEAGE SWITCH IN ACUTE LEUKAEMIA}

Chanukya Colonne ${ }^{1}$, Shalini Balendran ${ }^{1}$, Riana van der Linde $^{1}$, Ean Ooi $^{2}$, Elizabeth Tegg

${ }^{1}$ Haematology, Institute of Clinical Pathology and Medical Research (ICPMR), NSW Health Pathology, Westmead Hospital, Westmead, NSW, Australia; and ${ }^{2}$ Haematology, The Children's Hospital at Westmead, The Sydney Children's Hospitals Network, NSW Health, Westmead, NSW, Australia

Acute leukaemia is classified as myeloid, lymphoid, or of ambiguous lineage. ${ }^{1}$ Conversions of leukaemic cell lineage are rare, occurring in $<1 \%$ of childhood acute leukaemias. ${ }^{2}$ Historically, the vast majority of lineage conversions are described in childhood acute leukaemias following chemotherapy at relapse. ${ }^{3}$ Here, two cases of Philadelphia negative acute B-lymphoblastic leukaemia (B-ALL), with early lineage switch following pre-phase corticosteroid treatment, are presented. Both cases were negative for KMT2A rearrangements, a molecular aberration with an association for lineage conversions. $^{3}$

The first case is an 18-month-old boy, initially diagnosed with B-ALL that converted to an acute myeloid leukaemia (AML). He was treated with induction treatment for AML, however reverted to a B-ALL phenotype following one course of treatment. He was thereafter successfully treated with an ALLbased protocol. The second case, a 60-year-old woman, was also initially diagnosed with B-ALL and rapidly switched to an AML following corticosteroid treatment. She unfortunately succumbed to her disease. In both cases, the genetic signature of the leukaemia remained constant despite the phenotypic evolution.

Morphology, immunophenotyping, cytogenetics and molecular studies play a crucial role in the diagnosis of these rare lineage conversions, and accurate interpretation is essential to guide treatment and prognostication.

\section{References}

1. Swerdlow SH, Campo E, Harris NL, et al. WHO Classification of Tumours of Haematopoietic and Lymphoid Tissues. Lyon, France: IARC, 2008.

2. Rossi JG, Bernasconi AR, Alonso CN, et al. Lineage switch in childhood acute leukemia: an unusual event with poor outcome. Am J Hematol 2012; 87: 890-7.

3. Dorantes-Acosta E, Pelayo R. Lineage switching in acute leukemias: a consequence of stem cell plasticity? Bone Marrow Res 2012; 2012: 406796.

\section{IS THERE AN OPTIMAL TRANSFUSION STRATEGY FOR A CRITICALLY BLEEDING PATIENT?}

Michael Reade ${ }^{1,2}$

${ }^{1}$ Faculty of Medicine, University of Queensland, Brisbane, Qld, Australia; and ${ }^{2}$ Joint Health Command, Australian Defence Force, Canberra, ACT, Australia
Treatment of life-threatening, or 'critical' bleeding, has moved through several phases over the last 20 years, from a predominantly crystalloid or artificial colloid strategy, to one (based on flawed retrospective data) focussed on early use of a 1:1 ratio of red cell:plasma units (with the later contribution of platelets recognised), to a tailored approach based on the results of viscoelastic tests. Most recently, enthusiasm for stored or fresh whole blood is growing - which, curiously, was also the first approach to fluid resuscitation for bleeding. There is little doubt that the various current approaches reduce coagulopathy, morbidity and mortality compared to crystalloids or artificial colloids. However, evidence supporting one strategy over another is not as robust as many assume. Many retrospective observational studies are confounded by factors that influence the relationship between treatment and outcome, for which it is impossible to adjust using statistical methods alone. Surrogate measures of effectiveness, such as coagulopathy and biochemical parameters, do not always align with patient-centred outcomes. Randomised controlled trials are the only method of controlling for all possible confounders, observed and unobservable, but trials of a traditional design are extraordinarily difficult to conduct in this population. The solution might lie in new approaches to trial design, simultaneously evaluating testing and treatment, and building randomisation and outcome ascertainment into routine practice.

\section{MOLECULAR PROFILING IN HAEMATOLOGICAL MALIGNANCIES - THE MOST-LLY STUDY}

\author{
Steven Lane ${ }^{1,2}$ \\ ${ }^{I}$ QIMR Berghofer, Gordon and Jessie Gilmour Leukaemia \\ Research Laboratory, OIMR Berghofer, Herston, Old, \\ Australia; and ${ }^{2}$ Royal Brisbane and Women's Hospital, \\ Herston, Qld, Australia
}

MoST-LLy (Molecular Screening and Therapeutics in Leukaemia and Lymphoma) has a unique study design with molecular screening using Illumina's TruSight ${ }^{\mathrm{TM}}$ Oncology 500 Next Generation Sequencing (NGS) assay, and linking patients with advanced haematological malignancy with no standard of care treatment, to access targeted clinical trials and treatments they would otherwise not have access to.

The overarching MoST framework protocol governs molecular screening with each substudy reviewed and approved as an addenda to the program. We have developed two novel haematology specific treatment substudies with pharma that will soon open to recruitment, including:

- MoST 15: durvalumab + acalabrutinib for high-grade B cell lymphoma.

- MoST 16: PARP inhibitor for relapsed/refractory myeloid haematological malignancy.

MoST-LLy is the first molecular screening of its kind in blood cancers in Australia, and is being piloted across two sites initially with funding provided by the Leukemia Foundation of Australia 